\title{
Optimizing Steady Turns for Gliding Trajectories
}

\author{
Pedro F. A. Di Donato* \\ National Civil Aviation Agency - ANAC-Brazil, São José dos Campos SP 12246, Brazil \\ and \\ Ella M. Atkins \\ University of Michigan, Ann Arbor, Michigan 48109 \\ DOI: $10.2514 / 1 . G 000319$
}

\begin{abstract}
Dubins planners provide minimum-length planar paths between waypoints for turning constrained vehicles. For a no-thrust or glider aircraft, Dubins paths have been extrapolated to three dimensions by augmenting the planar path with a constant flight-path angle in each path segment. Best-glide flight maximizes the horizontal distance traveled per unit altitude loss, but steeper flight-path angles result in a maximum heading change per unit altitude loss. This paper investigates combinations of bank and flight-path angles yielding maximum ranges for gliding Dubins trajectories. Starting from the extrema flight-path angles usually applied in the literature, it is shown that the maximum range along each flight radial from an initial point (that is, the footprint) is obtained at a maximum glidepath angle for a given turn radius. This result is applied in a novel three-dimensional maximum-range Dubins path. Threedimensional Dubins examples with different heading constraints are presented. The impact of constraining solutions to a single pair of turning bank and flight-path angles is analyzed.
\end{abstract}

\section{Nomenclature}

\begin{tabular}{|c|c|c|}
\hline$A_{\text {model }}$ & $=$ & aircraft model \\
\hline$C_{D}$ & $=$ & drag coefficient \\
\hline$C_{D 0}$ & $=$ & zero-lift drag coefficient \\
\hline$C_{L}$ & $=$ & lift coefficient \\
\hline$C_{L \max }$ & $=$ & maximum lift coefficient \\
\hline$D$ & $=$ & drag force, lbf \\
\hline$d$ & $=$ & distance on horizontal plane, deg \\
\hline$g$ & $=$ & gravitational acceleration, $\mathrm{ft} / \mathrm{s}^{2}$ \\
\hline$h$ & $=$ & altitude, $\mathrm{ft}$ \\
\hline$k$ & $=$ & lift-induced drag coefficient \\
\hline$L$ & $=$ & lift force, $\mathrm{lbf}$ \\
\hline$l_{\mathrm{sc}}$ & $=$ & $\begin{array}{l}\text { critical Dubins path length for simply con- } \\
\text { nected reachable region, } n \text { mile }\end{array}$ \\
\hline$n$ & $=$ & load factor \\
\hline$R$ & $=$ & turn radius in the horizontal plane, $\mathrm{n}$ mile \\
\hline$R_{\min }$ & $=$ & minimum turn radius of Dubins path, $n$ mile \\
\hline$r_{N, i}, r_{E, i}, r_{D, i}$ & $=$ & $\begin{array}{l}\text { north, east, or down position of waypoint } i \\
\text { (north-east-down frame), n mile }\end{array}$ \\
\hline$S$ & $=$ & wing reference area, $\mathrm{ft}^{2}$ \\
\hline$V$ & $=$ & true airspeed, kt \\
\hline$W$ & $=$ & weight, lbf \\
\hline$\gamma$ & $=$ & flight-path angle, deg \\
\hline$\Delta \psi$ & $=$ & heading change over a turn, rad \\
\hline$\mu$ & $=$ & bank angle, deg \\
\hline$\xi$ & $=$ & flight radial, deg \\
\hline$\rho$ & $=$ & air density, $\operatorname{slug} / \mathrm{ft}^{3}$ \\
\hline$\psi$ & $=$ & heading angle, deg \\
\hline$\epsilon_{i}$ & $=$ & convergence tolerances \\
\hline
\end{tabular}

Received 30 November 2015; revision received 10 June 2016; accepted for publication 12 June 2016; published online 25 August 2016. Copyright $\odot$ 2016 by Pedro F. A. Di Donato. Published by the American Institute of Aeronautics and Astronautics, Inc., with permission. All requests for copying and permission to reprint should be submitted to CCC at www. copyright.com; employ the ISSN 0731-5090 (print) or 1533-3884 (online) to initiate your request. See also AIAA Rights and Permissions www.aiaa.org/ randp.

*Regulation Specialist (on leave); also Ph.D. Candidate, Aerospace Engineering Department, University of Michigan, Ann Arbor, MI 48109. Student Member AIAA.

Associate Professor, Aerospace Engineering Department. Associate Fellow AIAA.

\begin{tabular}{|c|c|c|}
\hline bd & $=$ & best-turn Dubins path condition \\
\hline bf & $=$ & best-turn footprint condition \\
\hline bg & $=$ & best-glide condition \\
\hline by & $=$ & $\begin{array}{l}\text { minimum altitude loss for a given turn heading } \\
\text { change }\end{array}$ \\
\hline $\begin{array}{l}C_{L \max } \\
\text { des }\end{array}$ & $\begin{array}{l}= \\
=\end{array}$ & $\begin{array}{l}\text { maximum lift coefficient condition } \\
\text { desired/given value }\end{array}$ \\
\hline $\max$ & $=$ & maximum value \\
\hline new & $=$ & new computed value \\
\hline old & $=$ & previous iteration value \\
\hline str & $=$ & wings-level condition \\
\hline & $=$ & maximum along radial $\xi$ \\
\hline $1,2,3$ & $=$ & $\begin{array}{l}\text { first, second, or third segment in the Dubins } \\
\text { path }\end{array}$ \\
\hline
\end{tabular}

\section{Introduction}

ENERAL aviation and unmanned fixed-wing aircraft typically $\widetilde{J}$ host a single engine or powerplant due to cost and weight considerations. Failure of this engine due to factors including fuel/ energy starvation or mechanical failure converts a powered aircraft into a glider. A typical single-engine aircraft experiencing loss of thrust does not have the ability to glide long term by methods such as thermalling. Loss of thrust therefore presents an urgent need for landing.

In 1957, L. E. Dubins published seminal work describing a lengthoptimal planar path between two points with prescribed position and tangents and a maximum constraint in average curvature [1]. The twodimensional Dubins path solution has been widely adopted for steered vehicles, and several extensions to it were proposed. Because aircraft also have turning constraints, the two-dimensional Dubins solution is a natural candidate to define a horizontal path connecting a headingconstrained initial state with a heading-constrained final state, e.g., a landing runway. Although a constant-altitude solution can directly apply the typical two-dimensional Dubins path, extensions are required to define the three-dimensional Dubins "airplane" path [2].

This paper investigates a question not yet carefully addressed in the Dubins airplane literature: What pair of turning bank and flight-path angles in a three-dimensional Dubins path provides the maximum gliding range for the overall path? To address this question, the paper first summarizes two solutions from the literature. One solution 
assumes a best-glide flight-path angle [3-ㅡㄹ, and the other minimizes altitude loss for a given heading change [6-9].

From this baseline, combinations of turn and straight segments to maximize the gliding footprint are explored, where the gliding footprint is defined as the ground-level region reachable by a gliding aircraft with no final heading constraint. The first contribution of this paper is an analysis that defines the gliding footprint problem in the established language of reachable sets $[10,11]$. The proposed conservative check guarantees that the footprint is simply connected. The second contribution of this paper is to expand $[3,4]$ with a footprint algorithm capable of computing the maximum-range turning bank and flight-path angle pair for each footprint flight radial. Results show that the previous solutions of best-glide [3, $\underline{4}]$ and minimum altitude losses for a given heading change are not optimal with respect to gliding range. The result is defined as the best-turn footprint. Moreover, this paper demonstrates that the maximum range is obtained with flight-path angles that are maximum for a given turn radius. This result is used to propose a more efficient algorithm for the best-footprint computation.

The final contribution of this paper is an algorithm to build a turnstraight-turn gliding path connecting a pair of heading-constrained two-dimensional positions such that the selected bank and flight-path angle pair for each turning segment maximizes the altitude at the final state. The resulting paths are defined as three-dimensional best-turn Dubins glider paths. This paper demonstrates that the maximum final altitude is also obtained with the maximum flight-path angle for a giving turn radius. A construction method for three-dimensional gliding paths is proposed to generate solutions with expanded reachability when compared with usual methods based on the best flight-path angle or minimum altitude loss for a given heading change.

This paper makes two primary assumptions. First, the results assume no wind is present. Second, only steady-state trajectory segments are modeled in proposed solutions. As a consequence, although each path segment respects an aircraft point-mass dynamics model, the proposed solutions assume airspeed can instantaneously change when transitioning between flight segments. Both assumptions have been made in related work. Steady-state trajectories allow fast guaranteed solutions that can be easily understood and followed by a pilot. The solutions can also be used to construct an initial estimate for an optimizer that can refine the proposed solution [12].

This paper is organized as follows. Section II presents the aircraft model and discusses the two approaches used by related work. Section III formulates the gliding footprint problem using a reachable set theory, presents the best-turn footprint algorithm, and compares the best-turn with the best-glide results. Section IV presents the bestturn three-dimensional Dubins planning algorithm and compares solutions with the best glide and minimum altitude loss over a given heading change, followed by conclusions in Sec. $\underline{\mathrm{V}}$.

\section{Background}

\section{A. Aircraft Model}

Throughout this study, trajectories are defined as sequences of "steady flight" segments. An equilibrium state does not experience acceleration over time. A steady flight condition is defined as an equilibrium condition at one altitude of the segment. All turns are considered coordinated, i.e., zero sideslip $(\beta=0)$, which results in the yaw angle always equal to the heading angle $\psi$. The flight parameters of true airspeed $V$, bank angle $\mu$, flight-path angle $\gamma$, horizontal turn radius $R$, and lift coefficient $C_{L}$ are constant for each steady flight state as given by equation of motion constraints $[\underline{13}, \underline{14}]$ :

$$
\begin{gathered}
-D-W \sin \gamma=0 \\
L \cos \mu-W \cos \gamma=0
\end{gathered}
$$

\footnotetext{
${ }^{\ddagger}$ Angle $\mu$ is usually not equal to the body frame Euler roll angle $\phi[13]$, which is the directly measured angle. Their difference, however, is small in most cases.
}

$$
L \sin \mu-\left(\frac{W V^{2} \cos ^{2} \gamma}{g R}\right)=0
$$

where $L$ is the lift force, $D$ is the drag force, $W$ is the weight, and $g$ is the gravitational acceleration. This work assumes flight within the standard envelope such that the aerodynamic forces are specified by the lift coefficient $C_{L}$ and coefficient of drag $C_{D}$, which is quadratic in $C_{L}$. With $C_{L}$ as a base parameter, no assumption regarding its relationship with the angle of attack is needed:

$$
\begin{aligned}
D & =\frac{1}{2} \rho(h) S C_{D} V^{2} \quad L=\frac{1}{2} \rho(h) S C_{L} V^{2} \\
C_{D}\left(C_{L}\right) & =C_{D 0}+k C_{L}^{2}
\end{aligned}
$$

For the preceding equations, $S$ is the wing reference area, $C_{D 0}$ is the zero-lift drag coefficient, and $k$ is the lift-induced drag coefficient. Air density $\rho$ is a function of altitude $h$ and based on U.S. Standard Atmosphere 1976 [15].

The flight-path angle does not depend on altitude. This fact allows the possible steady flight conditions to be expressed in terms of $(\gamma, \mu)$ pairs that are constant throughout each segment. Associated airspeed can be computed solving the following biquadratic equation:

$$
\left(\frac{C_{D 0} \rho(h) S}{2 W \cos \gamma}\right) V^{4}+(\tan \gamma) V^{2}+\left(\frac{2 k W \cos \gamma}{\rho(h) S \cos ^{2} \mu}\right)=0
$$

The turn radius and altitude loss during a turn are given by

$$
\begin{gathered}
R=\frac{V^{2} \cos \gamma}{g \tan \mu} \\
\Delta h=|\Delta \psi| R \tan \gamma=\frac{V^{2}|\Delta \psi| \sin \gamma}{g \tan \mu}
\end{gathered}
$$

Since this work is focused on maximum range and not minimum time, the true airspeed $V$ is only needed for radius $R$ calculations. $R$ is used for path planning, and its dependency on altitude is addressed considering a fixed value for each turn corresponding to the average value between the initial and final turn altitudes, as was done in [3] . In this work, $R$ associated with a given $(\gamma, \mu)$ pair is calculated using a simple three-step iterative algorithm:

1) Given an altitude, calculate the radius $R$ and associated altitude loss over a turn of angle $\Delta \psi$ with fixed radius $R$.

2) Update the altitude for the radius calculation using half the altitude loss calculated from the previous radius, limited to zero altitude.

3) Iterate over the new altitude until the altitude and radius converge.

Not all values of the flight-path and bank angles are feasible. Two physical limitations restrict their values: an aerodynamic limit represented by the maximum lift coefficient $C_{L \max } \underline{\S}$

$$
0 \leq C_{L} \leq C_{L \max }
$$

and a structural limit represented by the maximum load factor $n_{\text {max }}$ [13]. The latter is defined as a maximum value for the lift-weight ratio, from Eq. (2):

$$
n=\frac{L}{W}=\frac{\cos \gamma}{\cos \mu} \Rightarrow\left|\mu_{\max }\right|=\arccos \frac{\cos \gamma}{n_{\max }}<\arccos \frac{1}{n_{\max }}
$$

This study uses the performance data from a Cessna C172 provided in $[\underline{7,16]}$ for a flaps-up glide condition, as reproduced in Table 1 .

Therefore, this work conservatively assumes $|\mu| \leq 60 \mathrm{deg}$.

${ }^{\S}$ This work assumes $C_{L \text { min }}=0$ for numerical optimizations. This assumption does not affect the results because it is only associated with a highspeed limit. 
Table 1 Cessna 172 model data $[\underline{7,16}]$

\begin{tabular}{lccc}
\hline \hline Quantity & Symbol & Value & Unit \\
\hline Weight & $W$ & 2400 & lbf \\
Wing reference area & $S$ & 174 & $\mathrm{ft}^{2}$ \\
Zero-lift drag coefficient & $C_{D 0}$ & 0.037 & - \\
Lift-induced drag coefficient & $k$ & 0.0599 & - \\
Maximum lift coefficient & $C_{L \max }$ & 1.54 & - \\
Maximum load factor (flaps up) & $n_{\max }$ & 3.8 & - \\
\hline \hline
\end{tabular}

\section{B. Dubins Paths and Three-Dimensional Extensions}

The length-optimal planar path between two points with prescribed position and tangents given a maximum curvature constraint is composed of, at most, three segments of turn with minimum radius $(\mathrm{C})$ and straight lines (S), yielding solutions of type curve-straight-curve (CSC) or curve-curve-curve (CCC) [1] .

The pure extension of Dubins to three-dimensional space results in the inclusion of helicoidal arcs to the set of solutions for the twodimensional case [17]. Geometric solutions for CSC paths have been developed, but the path can result in a flight-path angle above the limits of the airplane, which is a problem that can be solved using numerical optimization techniques [18]. Another approach to three-dimensional extension is known as the Dubins airplane [2]. In this case, the curvature constraint is still considered only in the horizontal plane. New parameters (altitude and flight-path angle) are then added to the two-dimensional problem. Vertical variables are typically decoupled from horizontal state and control parameters. This model, therefore, contains flight-path angle constraints in its baseline formulation. Dubins airplane solutions depend on the altitude difference between initial and final points and the flight-path angle constraint.

The Dubins airplane is a purely geometric solution. To apply its solution to aircraft trajectory planning, a bridge between the geometry and the airplane dynamics must be constructed. The Dubins airplane requires constraints on the turn radius and flight-path angle. One proposed approach to link the Dubins solution to a speed profile defines a minimum turn radius given a fixed airspeed and limit bank angle using a small flight-path angle assumption [19]. Another approach builds the path as a sequence of steady flight conditions that respects dynamic constraints and presumes transitions between the steady flight conditions are feasible. This approach has been used for glider [3, $\underline{4}, \underline{6}-\underline{9}]$ and parafoil [5] applications. Note that [7,8] included accelerated segments to match airspeeds of different steady flight conditions.

For gliders and parafoils, smaller turning radii require steeper flight-path angles introducing a tradeoff between the turning path length and altitude drop over the turn. Figure 1 presents altitude loss when completing a full $\Delta \psi=360 \mathrm{deg}$ turn for different values of $(\gamma, \mu)$ pairs. For each $\mu$ value in Fig. 1 , possible $\gamma$ are constrained between two limits. The minimum constraint can be considered "hard" because it corresponds to points with a maximum lift

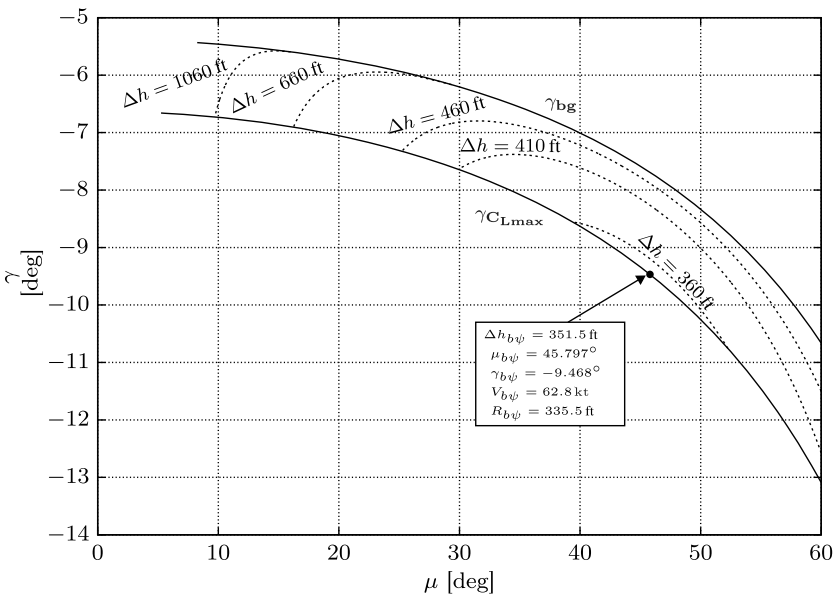

Fig. 1 Altitude loss $\Delta h$ over a complete $\Delta \psi=360 \mathrm{deg}$ turn for different $(\gamma, \mu)$ pairs. coefficient $C_{L \max }$. The higher limit corresponds to the best-glide flight-path angle $\gamma_{\mathrm{bg}}$ :

Definition 1: The best-glide flight-path angle $\gamma_{\mathrm{bg}}$ is the maximum or shallowest flight-path angle a gliding airplane can achieve for a given bank angle:

$$
\gamma_{\mathrm{bg}}(\mu)=\max \gamma
$$

subject to Eqs. (1- $\underline{-3})$ and (י) .

An analytic expression for $\gamma_{\mathrm{bg}}$ is given by

$$
\gamma_{\mathrm{bg}}=-\arctan \left(\frac{2 \sqrt{k C_{D 0}}}{\cos \mu}\right)
$$

This is a "soft" constraint because the airplane can perform steady turns with faster airspeeds, although high-speed turns are not relevant to this paper. All further results are thus restricted to flight-path angles $\gamma$ between the $\gamma_{C L \max }$ and $\gamma_{\mathrm{bg}}$ for each bank angle.

Instead of using best-glide flight-path angle, another group of researchers opted to use a different goal for its turn segments [6-9]:

Definition 2: The best-heading-change pair $\left(\gamma_{b \psi}, \mu_{b \psi}\right)$ is the $(\gamma, \mu)$ pair that results in minimum altitude loss for a given heading change $\Delta \psi$ :

$$
\left(\gamma_{b \psi}, \mu_{b \psi}\right)=\underset{\substack{\gamma \in\left[\gamma_{C L \max }, \gamma_{\mathrm{bg}}\right] \\ \mu \in[-60 \mathrm{deg}, 60 \mathrm{deg}]}}{\operatorname{argmin}} \frac{\Delta h}{\Delta \psi}=\underset{\substack{\gamma \in\left[\gamma_{C L \max }, \gamma_{\mathrm{bg}}\right] \\ \mu \in[-60 \mathrm{deg}, 60 \mathrm{deg}]}}{\arg \min } \frac{V^{2} \sin \gamma}{g \tan \mu}
$$

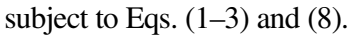

Using the small flight-path angle approximation, one can prove that the minimum loss of altitude with heading change is given exactly by $\mu_{b \psi}=45 \mathrm{deg}$ with $\gamma_{b \psi}=\gamma_{C L \max }$ []ㅡ. Assuming $\gamma_{b \psi}=$ $\gamma_{C L \text { max }}$ as in [7], but without using a small angle approximation, one can get!

$$
\cos ^{2} \mu_{b \psi}=\frac{1 \pm \sqrt{1-8\left[C_{D}\left(C_{L \max }\right) / C_{L \max }\right]^{2}}}{4}
$$

For the $\mathrm{C} 172$ model, $\left[C_{D}\left(C_{L \max }\right) / C_{L \max }\right]=0.1163$, and the only physically feasible root of Eq. (13) is $\mu_{b \psi} \approx 45.797 \mathrm{deg}$.

\section{Footprint and Reachable Sets}

The gliding footprint is defined as the ground-level region reachable by a gliding aircraft with no final heading constraint. Establishing a maximum-range footprint is a critical first step to emergency flight planning, even for solutions relying on visual recognition of emergency landing sites, as in [20]. Previous work has proposed footprint evaluation assuming a circle of arbitrary radius [21] or calculating the radius using a straight-flight best-glide angle with some margin [22]. Another approach $[3,4]$ is to compute paths composed by a turn with predefined bank angle and associated bestglide flight-path angle followed by a final approach straight segment with a wings-level best-glide flight-path angle.

The idea of a turn followed by a straight path is rooted in reachable sets obtained with Dubins planar paths with a free final heading $[10,11]$. Three important results are notable. First, footprint region boundaries are formed by paths composed of, at most, two segments, with each one being a minimum-radius turn arc or a straight segment. Second, for points outside the circles defined by simple minimumradius turns initiated at the origin point, the minimum length path is composed of a minimum-radius turn followed by a straight segment. Third, footprint regions for any path length above a critical value are simply connected. This critical value of the path length for simply connected regions $l_{\mathrm{sc}}$ is given by [10] as

\footnotetext{
TThe result presented here differs from [7] in the formulation of Eq. (3) by one instance of $\cos \gamma$ in the second term. The equations of motion used in this paper agree with $[13,14]$.
} 


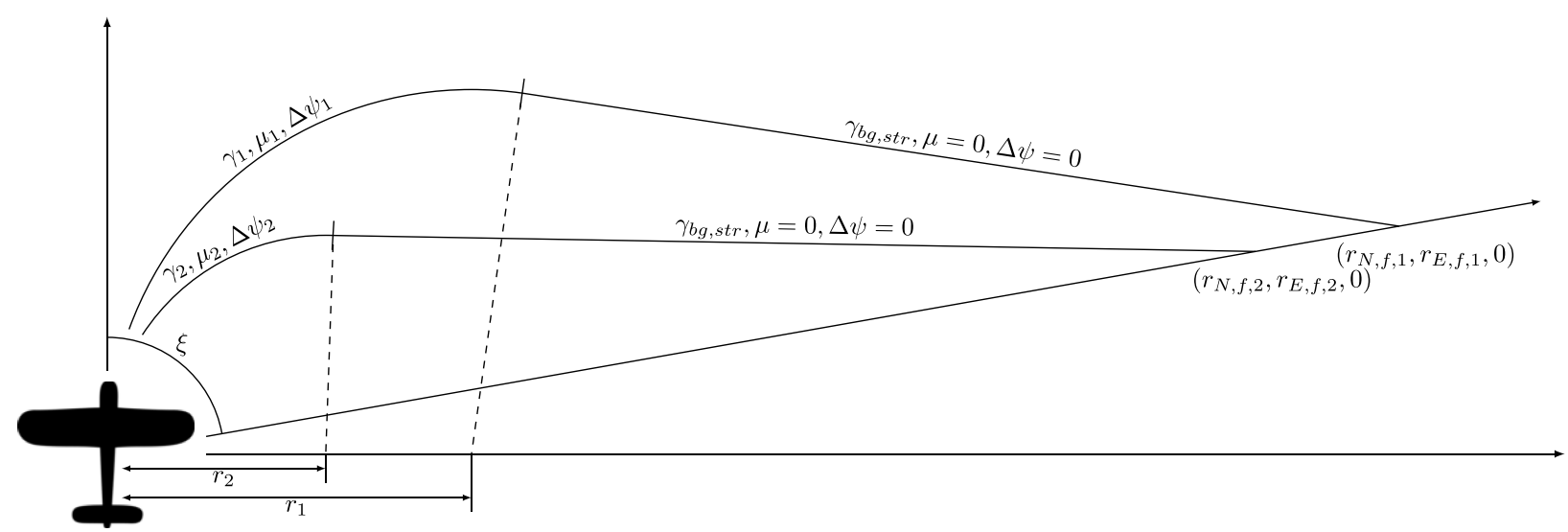

Fig. 2 Geometry of the footprint calculation problem.

$$
\frac{l_{\mathrm{sc}}}{R_{\min }}=2 \pi+\arccos \frac{23}{27} \approx 6.834
$$

where $R_{\min }$ is the minimum turn radius.

\section{Best-Turn Footprint Determination}

A footprint is defined as all coordinates or points on the ground that can be reached by an aircraft in flight with no final heading constraint [3]. Footprint extreme points are obtained with paths composed of a single turn followed by a straight path, as in $[3,4]$. As an extension of previous work, this paper presents an analysis of enclosed region connectivity. The main objective is to investigate whether the assumption made in $[\underline{3}, \underline{4}]$ of a best-glide turn per Definition 1 actually results in the largest possible footprint.

\section{A. Reachability Analysis}

Before analyzing best-turn footprints, this paper motivates the choice of paths composed of a turn followed by a straight segment. Assuming a constant flight-path angle for each flight condition and one simple minimum turn radius, the airplane footprint computation essentially maps to the reachable set for a Dubins car with a prescribed path distance and unconstrained final heading. The previous results for this problem discussed in Sec. II.C reveal that extremal Dubins paths of sufficient lengths take the turn-straight segment form. Moreover, these Dubins results guarantee a simply connected reachable region. Using conservative values of the flight-path angle and turn radius for the $\mathrm{C} 172$, one can estimate the minimum above ground level (AGL) altitude for which the simply connected assumption is assured. As an example, with $R_{\min }=1000 \mathrm{ft}$ and $\gamma=-10 \mathrm{deg}$, Eq. (14) results in a minimum AGL altitude of approximately $1205 \mathrm{ft}$. For initial altitudes above this value, the footprint boundary can be calculated by considering only paths composed of an initial turn followed by a straight segment, without risk of unreachable interior points.

\section{B. Maximum-Range Footprint Algorithm}

A footprint is defined from an initial state $\left(x_{0}, y_{0}, z_{0}, \psi_{0}\right)$ with consideration of the aircraft performance envelope. The design parameters are the turn and straight-flight steady flight states. Reachable points are obtained from an initial turn over heading change $\Delta \psi$ followed by a straight-flight segment at straight-flight best-glide flight-path angle $\gamma_{\mathrm{bg}, \mathrm{str}}$ to zero AGL altitude. This approach does not include effects of transitioning between steady flight conditions.

Figure 2 illustrates the proposed problem. For each $(\gamma, \mu)$ pair, one associated turn radius $R$ is defined, and there can be one arc with angle $\Delta \psi$ that results in the final path point with zero AGL altitude along a radial $\xi$ relative to the initial location. ${ }^{* *}$ The problem of finding the best-turn footprint reduces to the problem of finding, for

**Depending on the problem, there can be zero, one, or two values of $\Delta \psi$ that satisfy this condition. This paper only uses the $\Delta \psi$ with a smaller value, resulting in the greatest range (see Proposition 1 proof). each radial $\xi$, the triple $(\gamma, \mu, \Delta \psi)$ that results in the final path point along radial $\xi$ with the maximum distance from the initial point. This is performed by calculating for each pair $(\gamma, \mu)$ the required $\Delta \psi$, resulting in a final point over the radial $\xi$, and selecting the triple that maximizes range. This problem gives rise to the following definition:

Definition 3: The best-turn footprint pair $\left(\gamma_{\mathrm{bf}}, \mu_{\mathrm{bf}}\right)$ is the $(\gamma, \mu)$ pair associated with each flight radial $\xi$ that, when used for the turn segment of a path composed of a turn followed by a wings-level bestglide $\gamma_{\text {bg,str }}$ segment to the ground, results in the final point over that radial with the largest distance from the initial point:

$$
\left(\gamma_{\mathrm{bf}}, \mu_{\mathrm{bf}}\right)=\underset{\substack{\gamma \in\left[\gamma_{C L \max }, \gamma_{\mathrm{bg}}\right] \\ \mu \in[-60 \mathrm{deg}, 60 \mathrm{deg}]}}{\arg \max }\left\|\left[r_{N f}(\gamma, \mu), r_{E f}(\gamma, \mu)\right]\right\|
$$

subject to Eqs. (1- $\underline{3})$ and ( $\underline{8})$, where $\left(r_{N f}, r_{E f}\right)$ is the end point over radial $\xi$ as per Fig. $\overline{2}$.

For each value of $\bar{\xi}$, a brute-force search over all values of $(\gamma, \mu)$ can be executed to find $\left(\gamma_{\mathrm{bf}}, \mu_{\mathrm{bf}}\right)$. Figure $\underline{3}$ presents a pair of optimal footprints for an initial altitude of $2000 \mathrm{ft}$. The initial condition is at the origin with heading zero (north). Best-turn bank angle $\mu_{\mathrm{bf}}$ contours are presented as straight lines. Figure 4 presents the calculated optimal bank and flight-path angles for each radial $\xi$. Additionally, limits are plotted for each bank angle associated with the flight-path angle curves.

The final footprint is close to a circle. This is because the chosen altitude is sufficiently high that the effects of altitude loss during the

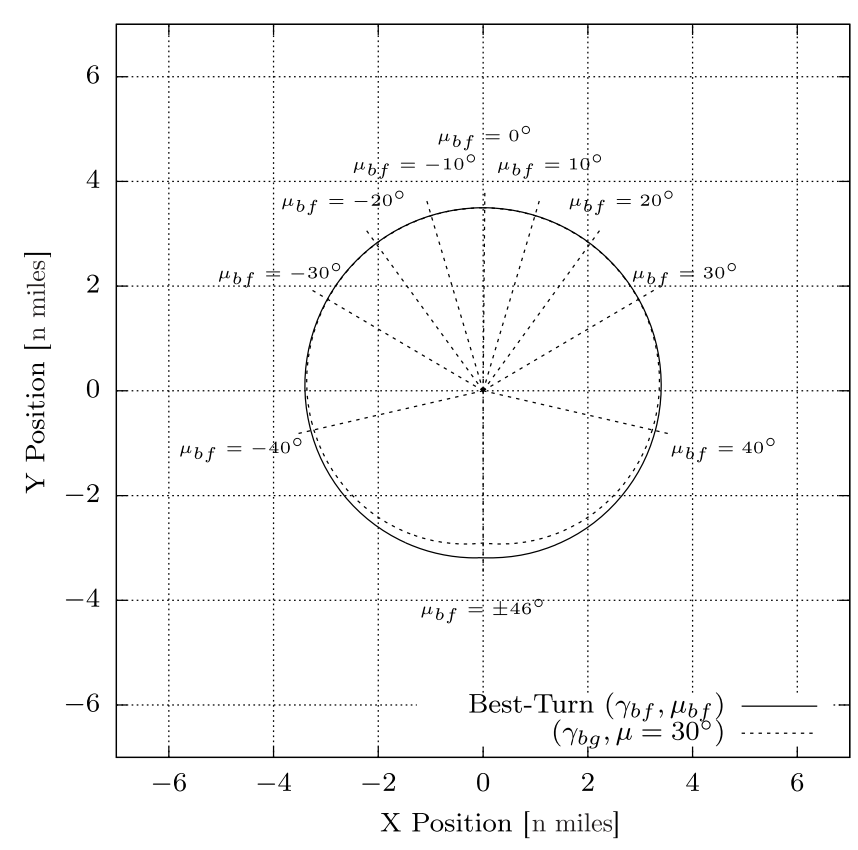

Fig. 3 Optimal footprints from an initial altitude of $2000 \mathrm{ft}$ highlighting selected bank angles. 

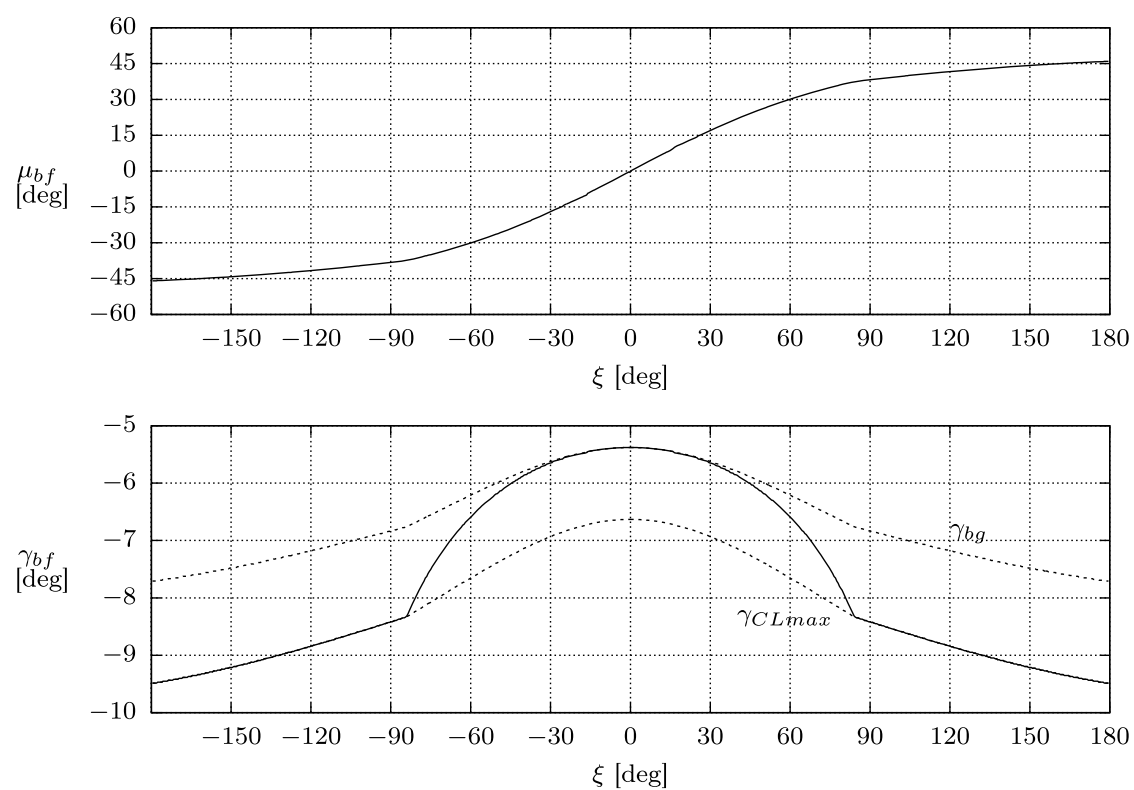

Fig. 4 Best-turn bank and flight-path angles for different radial flight directions.

initial turn with respect to the best straight glide are minimal. The reachable distance in the case of straight flight $(\xi=0 \mathrm{deg})$ is, of course, greater than in the case of opposite direction $(\xi=180 \mathrm{deg})$ flight. Note that the optimal bank angle increases from zero for the straight-flight direction up to $\mu=46 \mathrm{deg}$ for $\xi=180 \mathrm{deg}$. Moreover, Fig. 4 shows that the optimal flight-path angle also varies from the best-glide flight-path angle to the limit associated with $C_{L \max }$.

These results are a direct consequence of turn radius dependency on flight-path angle. For radial directions substantially different from initial heading, the gain in distance associated with the reduction in the turn radius compensates for the steeper gradient. The maximum optimal bank angle computed is $\mu=46 \mathrm{deg}$, consistent with the results of Sec. II.B. Figure 3 presents as a dashed line the footprint computed with a standard rate best-glide turn $\left(\gamma_{\mathrm{bg}}, \mu=30 \mathrm{deg}\right)$ to highlight the benefit of using the computed best-turn parameters. As expected, higher gains are observed for radials that require larger heading changes.

Although a brute-force search provides maximum range for the general case, an interesting result can be deduced specifically for the nowind case. This result reduces the two-dimensional search for the best $(\gamma, \mu)$ pair to a one-dimensional search for the best-turn radius, significantly increasing the speed of the algorithm. As a first step, a new definition is proposed:

Definition 4: The best-glide-for-turn-radius pair $\left[\gamma_{b r}(R, h), \mu_{b r}(R, h)\right]$ is the $(\gamma, \mu)$ pair associated with turn radius $R$ and altitude $h$ that result in the maximum (shallowest) flight-path angle in the turn. Mathematically,

$$
\mu_{b r}(R, h)=\underset{\mu \in[0 \mathrm{deg}, 60 \mathrm{deg}]}{\arg \max } \gamma(\mu, R, h)
$$

subject to Eq. (

Note that $\gamma(\bar{\mu}, R, h)$ is implicitly defined by the following equality constraint [obtained from Eqs. (ㅁ) and (ㅁ)]:

$$
\left(\frac{C_{D 0} \rho(h) S g^{2} R^{2} \tan ^{2} \mu}{2 W \cos ^{3} \gamma}\right)+\left(\frac{g R \tan \mu \tan \gamma}{\cos \gamma}\right)+\left(\frac{2 k W \cos \gamma}{\rho(h) S \cos ^{2} \mu}\right)=0
$$

Furthermore,

$$
\gamma_{b r}(R, h)=\gamma\left(\mu_{b r}, R, h\right)
$$

Optimization tools (in MATLAB) were used to build a best-glidefor-turn-radius database with $\left[R, h, \gamma_{b r}(R, h), \mu_{b r}(R, h)\right]$ quadruples.
Results were obtained for a database with $R \in[225,6000] \mathrm{ft}$, resolution of $\Delta R=5 \mathrm{ft}$ and $h \in[0,2000] \mathrm{ft}$, and resolution of $\Delta h=200 \mathrm{ft}$.t⿱亠䒑 Figure $\underline{5}$ presents lines connecting $(\mu, \gamma)$ of such quadruples for different radii. Each continuous line corresponds to an altitude, and constant radius solutions are represented by dashed lines. Data points represent the same value of radius. Note that, although the altitude influences the position of the points over each line, both lines are superimposed. Also observe that the lines are close to $\gamma_{\mathrm{bg}}$ for low values of $\mu$ and close to $\gamma_{C L \text { max }}$ for large values of $\mu$. In fact, a simple optimization of Eq. (16) can return values that correspond to $C_{L}$ values above $C_{L \text { max }}$, i.e., the point below the line $\gamma_{C L \text { max }}$ of Fig. 5 . In this case, the point is discarded and a quadruple corresponding to the intersection of a constant radius line and $\gamma_{C L \text { max }}$ line is used.

Using the definition of $\left[\gamma_{b r}(R, h), \mu_{b r}(R, h)\right]$, the footprint problem is simplified by the following proposition:

Proposition 1: Given a turn radius $R$, initial altitude $h$, and radial $\xi$, the maximum range over $\xi$ from $h$ in the no-wind case is achieved with a maximum turning flight-path angle for $R$, i.e., $\left(\gamma_{\mathrm{bf}}, \mu_{\mathrm{bf}}\right)=$ $\left[\gamma_{b r}(R, h), \mu_{b r}(R, h)\right]$ 望

Proof: Figure $\underline{6}$ revisits the footprint calculation problem, illustrating angles $\bar{\xi}$ and $\Delta \psi$. Consider the relation between these two angles (flight-path angles during the turn $\gamma_{\operatorname{trn}}$ and straight flight $\gamma_{\mathrm{str}}$ ), $R$, and $\Delta h=-r_{D_{i}}$ :

$$
\Delta h=\Delta h_{\mathrm{trn}}+\Delta h_{\mathrm{str}}
$$

$$
=R \Delta \psi \tan \gamma_{\mathrm{trn}}+d_{\mathrm{str}} \tan \gamma_{\mathrm{bg}, \mathrm{str}}
$$

$$
=R \Delta \psi \tan \gamma_{\mathrm{trn}}+R\left(1-\frac{\cos \xi}{\cos (\Delta \psi-\xi)}\right) \cot (\Delta \psi-\xi) \tan \gamma_{\mathrm{bg}, \mathrm{str}}
$$

From Fig. 6, note that $\Delta \psi>\xi$; and from Eq. (21), $\Delta \psi=2 \xi \Rightarrow \Delta h_{\text {str }}^{-}=0$. Thus,

$$
\xi<\Delta \psi<2 \xi
$$

\footnotetext{
${ }^{\dagger}$ Higher values of radius $R$ lead to numerical issues during optimization. ${ }^{\sharp}$ Note that $\left[\gamma_{b r}(R, h), \mu_{b r}(R, h)\right]$ depend on the average altitude of the turn (see Sec. II.A), which is associated with the initial altitude $h$.
} 


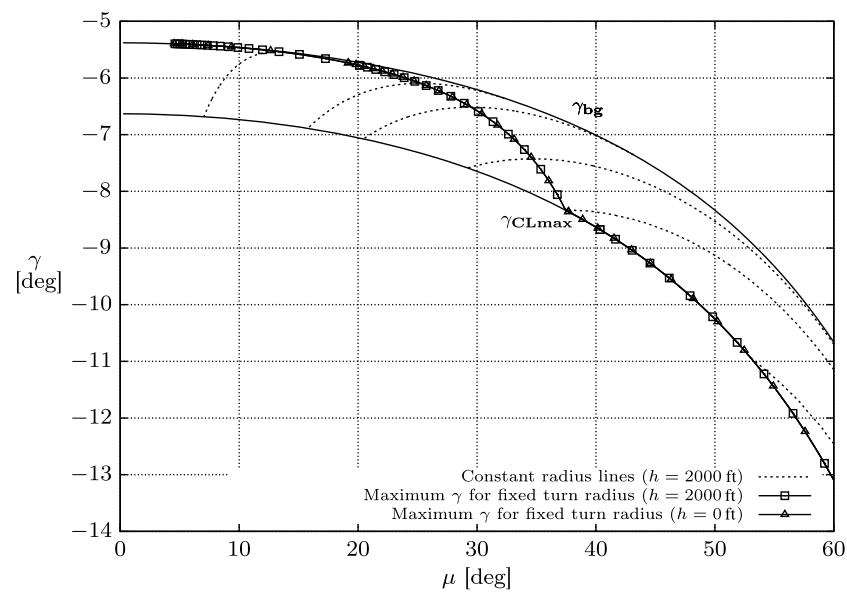

Fig. 5 Best-glide-for-turn-radius pairs $\left[\gamma_{b r}(R, h), \mu_{b r}(R, h)\right]$ for the Cessna 172 model.

The total distance along $\xi$ is given by

$d_{\xi}=R\left[\sin \xi+\cos \xi \tan (\Delta \psi-\xi)+\left(1-\frac{\cos \xi}{\cos (\Delta \psi-\xi)}\right) \frac{1}{\sin (\Delta \psi-\xi)}\right]$

Taking the derivative with respect to $\Delta \psi$,

$$
\begin{gathered}
\frac{\partial d_{\xi}}{\partial \Delta \psi}=R\left[\cos \xi\left(\tan ^{2}(\Delta \psi-\xi)+1\right)-\frac{\cos \xi}{\cos ^{2}(\Delta \psi-\xi)}\right. \\
\left.+\frac{\cos (\Delta \psi-\xi)(\cos \xi / \cos (\Delta \psi-\xi)-1)}{\sin ^{2}(\Delta \psi-\xi)}\right] \\
=R\left[\frac{\cos \xi-\cos (\Delta \psi-\xi)}{\sin ^{2}(\Delta \psi-\xi)}\right]
\end{gathered}
$$

and given that $\xi<\pi$,

$$
\forall \Delta \psi: \xi<\Delta \psi<2 \xi \Rightarrow \frac{\partial d_{\xi}}{\partial \Delta \psi}<0
$$

For a gliding airplane assuming $\gamma_{\mathrm{str}}=\gamma_{\mathrm{bg}, \mathrm{str}}, \gamma_{\mathrm{trn}} \leq \gamma_{\mathrm{str}}<0$. Thus, taking the limit of Eq. (21) gives

$$
\forall \xi>0 \quad \lim _{\Delta \psi \rightarrow \xi} \Delta h=-\infty
$$

The derivative of Eq. (21) with respect to $\gamma_{\text {trn }}$ yields:

$$
\begin{gathered}
\frac{\partial \Delta h}{\partial \gamma_{\mathrm{trn}}}=R \Delta \psi\left(\tan ^{2} \gamma_{\mathrm{trn}}+1\right)=\frac{R \Delta \psi}{\cos ^{2} \gamma_{\mathrm{trn}}} \\
\therefore \forall \Delta \psi>\xi>0 \quad \frac{\partial \Delta h}{\partial \gamma_{\mathrm{trn}}}>0
\end{gathered}
$$

Note that $\Delta h$ as defined is always negative for a gliding airplane. Equation (29) states that higher glide angles (shallower $\gamma$ ) will result in less loss of altitude $\Delta h$ for the same horizontal path projection. Suppose $R, \gamma_{\text {str }}$, and $\xi$ are fixed and for a certain $\gamma_{\text {trn,1 }}$. The maneuver with $\Delta \psi_{1}$ results in $\Delta h_{1}$ and a distance along the radial $d_{\xi, 1}$. Moreover, suppose there exists a $\gamma_{\mathrm{trn}, 2}>\gamma_{\mathrm{trn}, 1}$ capable of producing the same $R$. From Eq. (29), for the same $\Delta \psi_{2}=\Delta \psi_{1}, \Delta h_{2}>\Delta h_{1}$, i.e., less altitude is lost to reach the flight radial at the same point. But, from Eq. (27), $\exists \Delta \psi_{2, \text { opt }}<\Delta \psi_{1}$ such that $\gamma_{\text {trn,2 }}$ results in $\Delta h_{2, \text { opt }}=$ $\Delta h_{1}$; and from Eq. (26), $d_{\xi, 2, \mathrm{opt}}>d_{\xi, 1}$. Thus, any flight-path angle other than $\gamma_{b r}(R, h)$ will result in a smaller range along a radial than the range provided by any $\gamma_{b r}(R, h)$ for the same $\Delta h$.

Instead of searching over all $(\gamma, \mu)$ pairs for the best range, it is sufficient to search through possible $R$ with associated $\left[\gamma_{b r}(R, h), \mu_{b r}(R, h)\right]$. Algorithm 1 outputs the maximum possible distance along the given radial $d_{\xi}^{-}$as well as $R_{\mathrm{bf}}$ and the associated $\left(\gamma_{\mathrm{bf}}, \mu_{\mathrm{bf}}\right)$ pair per Definition 4.

Algorithm 1 receives as input the aircraft model $A_{\text {model }}$, the initial position and heading $\left(r_{N, 0}, r_{E, 0}, r_{D, 0}, \psi_{0}=0\right)$, and the turn radius range $\left[R_{\min }, R_{\max }\right]$. The algorithm restrictions $\psi_{0}=0$ and $0 \mathrm{deg}<$ $\xi_{\text {des }} \leq 180$ deg are used for simplicity considering the symmetry of the no-wind case. The algorithm first selects an $R$ from a user-defined range and associated $(\gamma, \mu)$ pair from the precomputed database. Then, an initial turnover $\Delta \psi$ is chosen, and the trajectory to zero AGL altitude is computed. The flight radial $\xi$ relative to the final position is evaluated. Then, $\Delta \psi$ is updated using a variable-step hill-climbing method. The initial heading change step size is set to $10 \mathrm{deg}$. This step size is divided by 10 each time the desired radial is crossed or a local maximum $\xi$ is reached. This procedure is repeated until $\xi$ converges to $\xi_{\text {des }}$ or when $\xi$ converges to a maximum that is inferior to $\xi_{\text {des }}$. Note that, for some $R$ and initial altitude values, a negative altitude may be obtained during the turn segment with minimum heading change $\Delta \psi=\xi_{\text {des }}$. In such cases, $\xi_{\text {des }}$ is not reachable. Functions Position After Turn and Position After Straight Glide UpTo Ground calculate position change.

Figure 7 revisits Fig. 5, with the results obtained from the bruteforce search over $\gamma$ and $\mu$ overlaid. Those are the same results

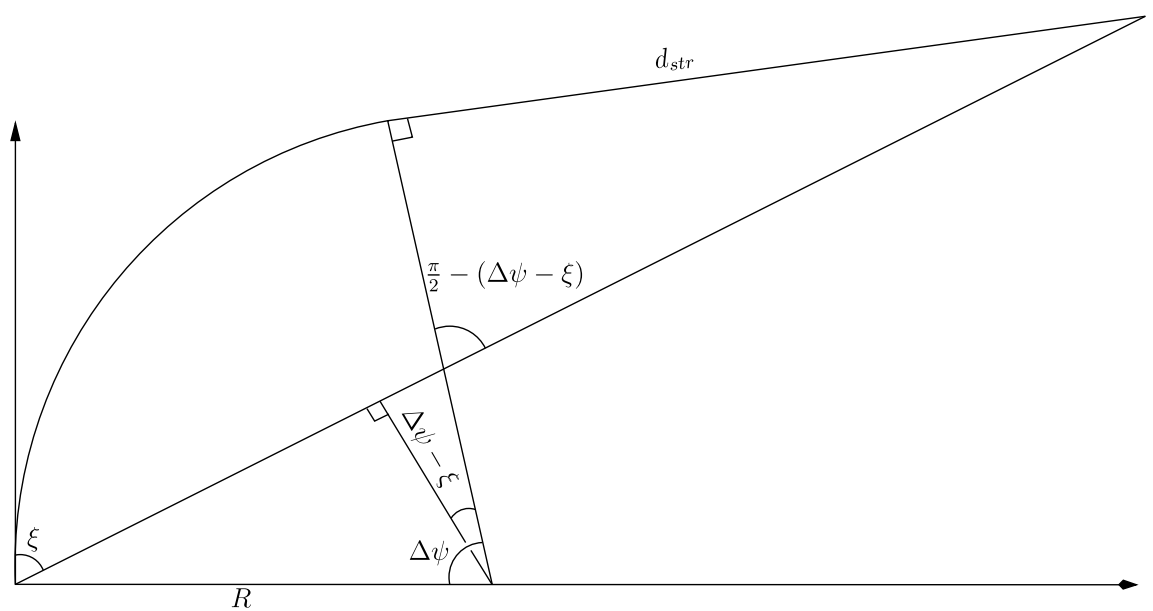

Fig. 6 Relevant angles for the footprint calculation problem. 


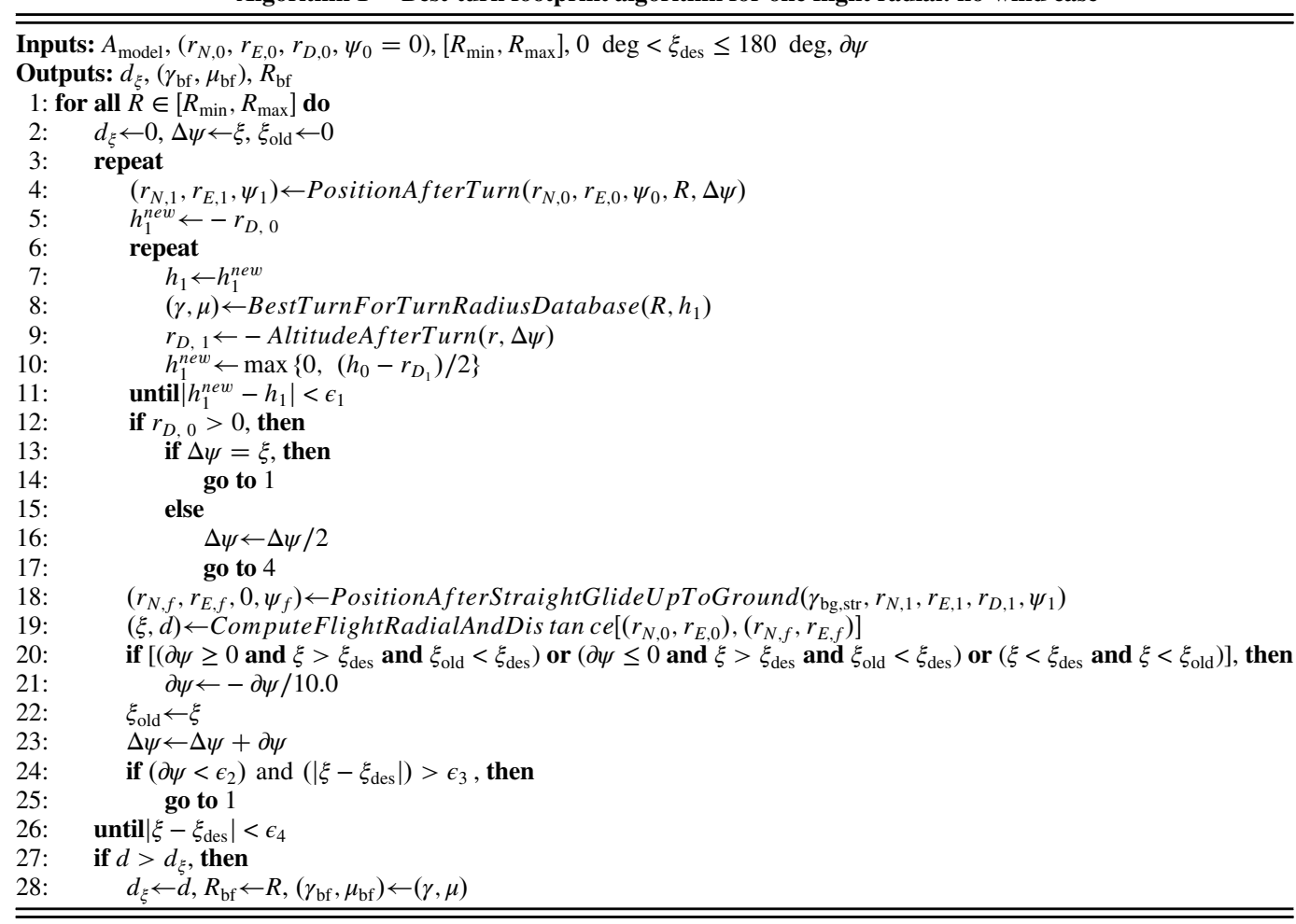

presented in Figs. 3 and 4 . Note in Fig. 7 the agreement between results for the no-wind case with $\left[\gamma_{b r}(R, h), \mu_{b r}(R, h)\right]$, which further confirms Proposition 1.

Figure $\underline{8}$ presents the calculated optimal turn radius and bank angle for each radial $\xi$ using Algorithm 1 . Note that results are limited to the previously computed $\left[\gamma_{b r}(R, h), \bar{\mu}_{b r}(R, h)\right]$ database.

\section{Best-Turn Dubins Glider Paths}

An important application of this work is to increase the gliding range of Dubins airplane solutions relative to previous work through the use of best-turn profiles. Since the original Dubins airplane [2] did not correlate the flight path with turning performance, this work introduces the term Dubins glider for paths composed of steady turn and straight segments that satisfy glider equations (1) to (3) and $C_{L \max }$ and maximum load factor restrictions. This work focuses on illustrating possible altitude savings so that final altitude is left free to facilitate the analysis of this savings. The objective of the maximum-range Dubins solver is then to select the bank and flight-path angle pair associated

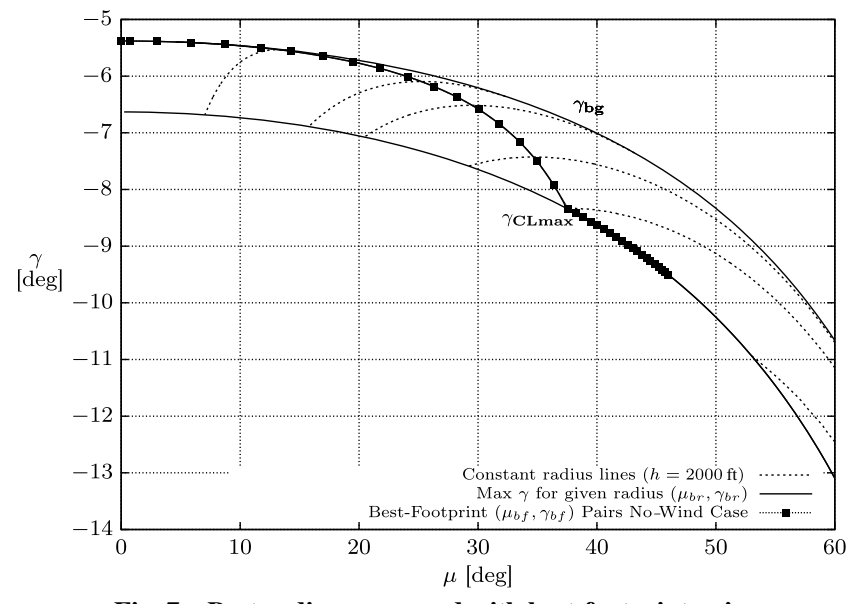

Fig. 7 Best radius compared with best-footprint pair. with each turning segment that results in minimum altitude loss. Results are analyzed by comparing the final altitude to that obtained with the two strategies previously proposed: a Dubins solver assuming best-glide flight-path angle over turns as well as straight segments, and a Dubins solver assuming best-heading-change turns and best-glide flight-path angles for straight segments.

A Dubins glider path is composed of, at most, three segments in a curve-straight-curve sequence. Dubins' original solution [1] included a curve-curve-curve sequence not used in this work. CCC sequences are not possible when there is a large distance between the initial and final points, and they add significant complexity to the problem because a new radius must also be considered. Their consideration, useful mostly when initial and final position are very close to each other, is left for future work. This section associates subscripts 1, 2, and 3 with each Dubins glider path segment. Since the goal is to find a path that results in minimum altitude loss, straight segments are achieved with a wings-level best-glide steady flight state $\left(\gamma_{\mathrm{bg}, \mathrm{str}}, \mu=0\right)$. For turn (curved) segments, however, the Dubins airplane [2] minimum turn radius solution cannot be directly applied.

Figure 9 illustrates and motivates the definition of the maximum-range glider path planner. A vertical projection of two trajectories is shown from the origin to the point $(0.5 \mathrm{n}$ mile, $0.5 \mathrm{n}$ mile $) \approx(3038 \mathrm{ft}, 3038 \mathrm{ft})$ given initial heading $\psi_{i}=0 \mathrm{deg}$ and final heading $\psi_{f}=90 \mathrm{deg}$. One of the trajectories is computed with the pair $\left(\mu_{1}=\mu_{3}=30 \mathrm{deg}\right.$, $\left.\gamma_{1}=\gamma_{3}=\gamma_{\mathrm{bg}} \approx-6.20 \mathrm{deg}\right)$ representing a standard best-glide turn with $R=962 \mathrm{ft}$ and $V=79.4 \mathrm{kt}$ at an altitude of $2000 \mathrm{ft}$, and the other with $\left(\mu_{1}=\mu_{3}=60 \mathrm{deg}, \gamma_{1}=\gamma_{3}=\gamma_{C L \max } \approx-13.09 \mathrm{deg}\right)$, which results in $R=272 \mathrm{ft}$ and $V=73.9 \mathrm{kt}$ at the same $2000 \mathrm{ft}$ altitude. The wings-level best-glide flight-path angle for both trajectories is $\gamma_{2}=$ -5.38 deg with $V=74.0 \mathrm{kt}$.

Figure 9 a illustrates trajectory differences reflecting the fact that an increase in bank angle and reduction in flight-path angle results in a reduced turn radius, and a consequently reduced total horizontal path distance. However, the right graph of the same figure shows that the reduced total path distance does not result in a smaller altitude loss. This is a consequence of the steeper flight-path angle than best glide.

This work approaches the compromise between turn radius and altitude loss for a gliding path (Fig. 9) using the following proposition, which is analogous to that applied to the footprint problem. 

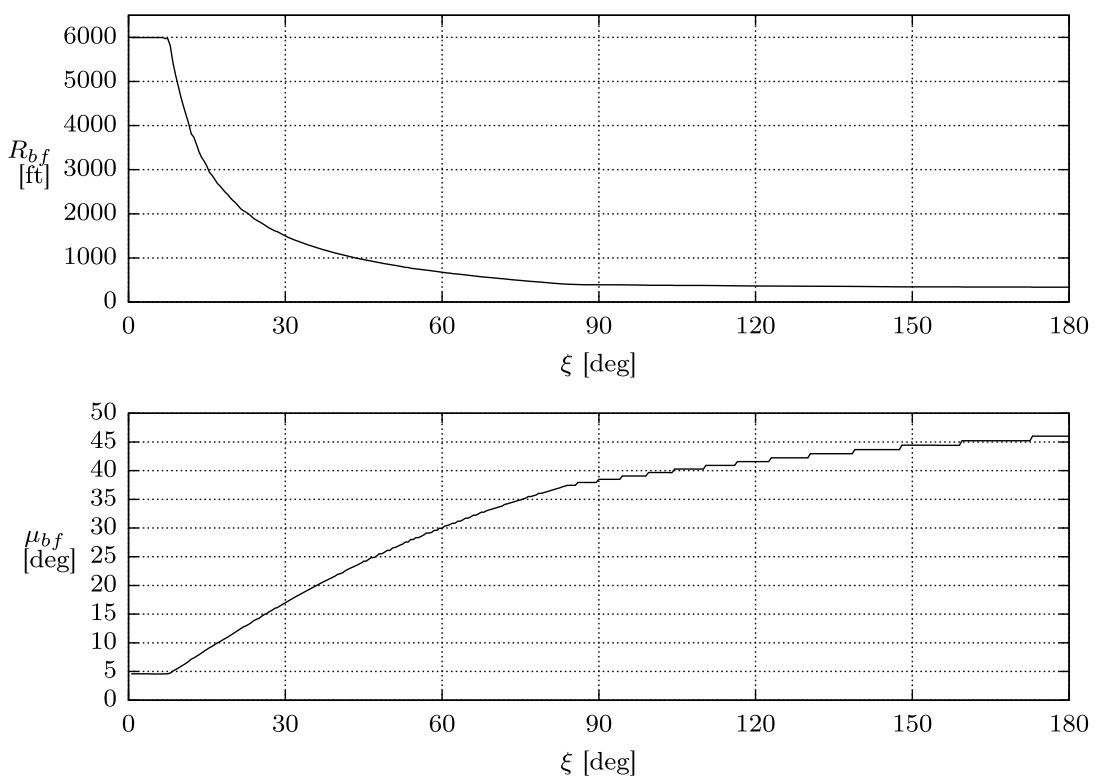

Fig. 8 Best-turn radius and bank angles for different radial flight directions.

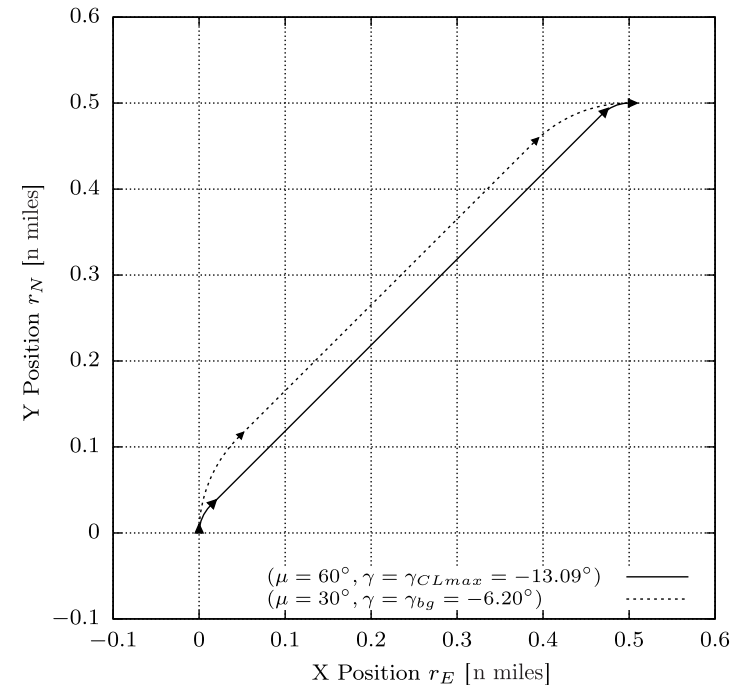

a)

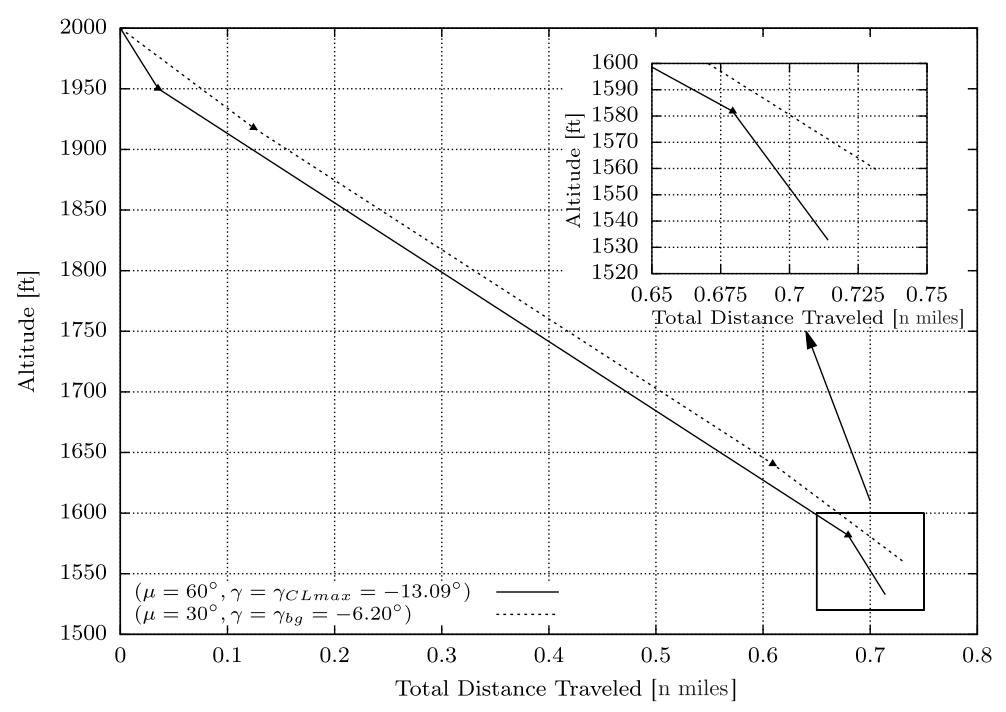

b)

Fig. 9 Comparison of two different trajectories with the same initial and final conditions. .

Proposition 2: For a Dubins glider given no wind, maximum altitude at the final point is achieved with a maximum turning flightpath angle for a specific turn radius, i.e., $\left[\gamma_{b r}(R, h), \mu_{b r}(R, h)\right]$.

Proof: Dubins glider path horizontal projections are normal Dubins two-dimensional paths. Such paths are completely determined by twodimensional initial and final positions and heading and turning radii. Given a fixed two-dimensional path, the minimum altitude loss is obtained using the minimum flight-path angle, i.e., the shallowest in each of its segments. For curved segments, each angle is obtained using $\left[\gamma_{b r}(R, h), \mu_{b r}(R, h)\right]$ by its definition.

Algorithm 2 summarizes Dubins glider path construction for a given turn radius $R_{i}$ for each segment $i$, aircraft model $A_{\text {model }}$, initial position and heading $\left(r_{N, 0}, r_{E, 0}, r_{D, 0}, \psi_{i}\right)$, and final position and heading with free altitude $\left(r_{N, f}, r_{E, f}, \psi_{f}\right)$. This algorithm returns the three-dimensional Dubins glider trajectory traj3D and final altitude $r_{D, 3}$. A low-complexity iterative method finds each turn altitude. Analogous to the Sec. II.A method, each turn is computed with a given turn radius and a flight-path angle for the average altitude between the turn segment's initial and final values. Linear interpolation is used between the closest values of altitude presented in the database. To determine the best-turn Dubins glider path, all turn radius values of the best-turn Dubins glider database are tested. This approach extends previous work that defines turns with either a best-glide flight-path angle or best-turn-for-heading-change condition. Note that some Dubins paths may not exist. This work uses the following definition for representing the results of this optimization:

Definition 5: The best-turn Dubins glider radii $\left(R_{\mathrm{bd}, 1}, \infty, R_{\mathrm{bd}, 3}\right)$ represent the turn radii for the segments of the Dubins glider path resulting in the maximum final altitude for given airplane model, initial position and heading, and final position and heading with free altitude. These best-glide-for-turn-radius pairs are denoted best-turn Dubins glider pairs $\left[\left(\gamma_{\mathrm{bd}, 1}, \mu_{\mathrm{bd}, 1}\right),\left(\gamma_{\mathrm{bd}, 3}, \mu_{\mathrm{bd}, 3}\right)\right]$.

The final heading constraint further increases the complexity of the problem when compared with the footprint computation in Sec. III. To illustrate optimal solution characteristics, best-turn paths are computed for a series of final positions covering a $4 \mathrm{n}$ mile lateral region. Since the required heading change between the initial and final $\Delta \psi$ can significantly affect the trajectory, the final heading is fixed for each graph, which is an approach common in the literature [23]. Plots are presented with the usual aircraft standard of a zero heading pointing up and increasing clockwise. The initial condition is the same as in Sec. III: aircraft at the origin heading north with an altitude of $2000 \mathrm{ft}$. 
Algorithm 2 Dubins gliding algorithm: no-wind case

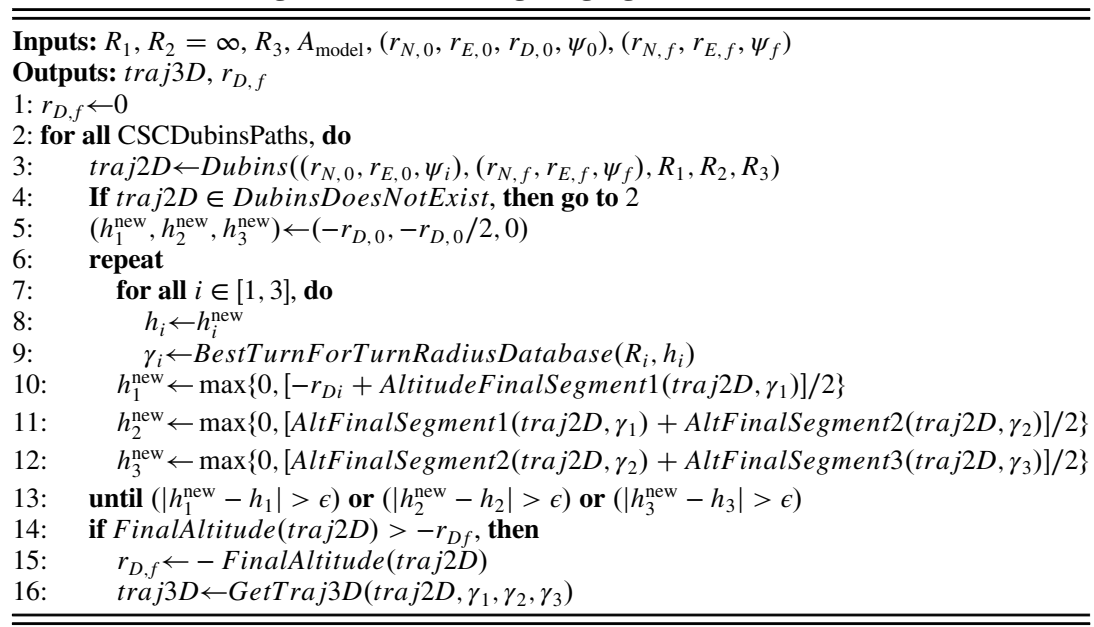

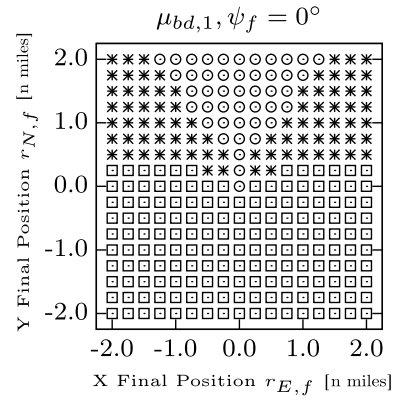
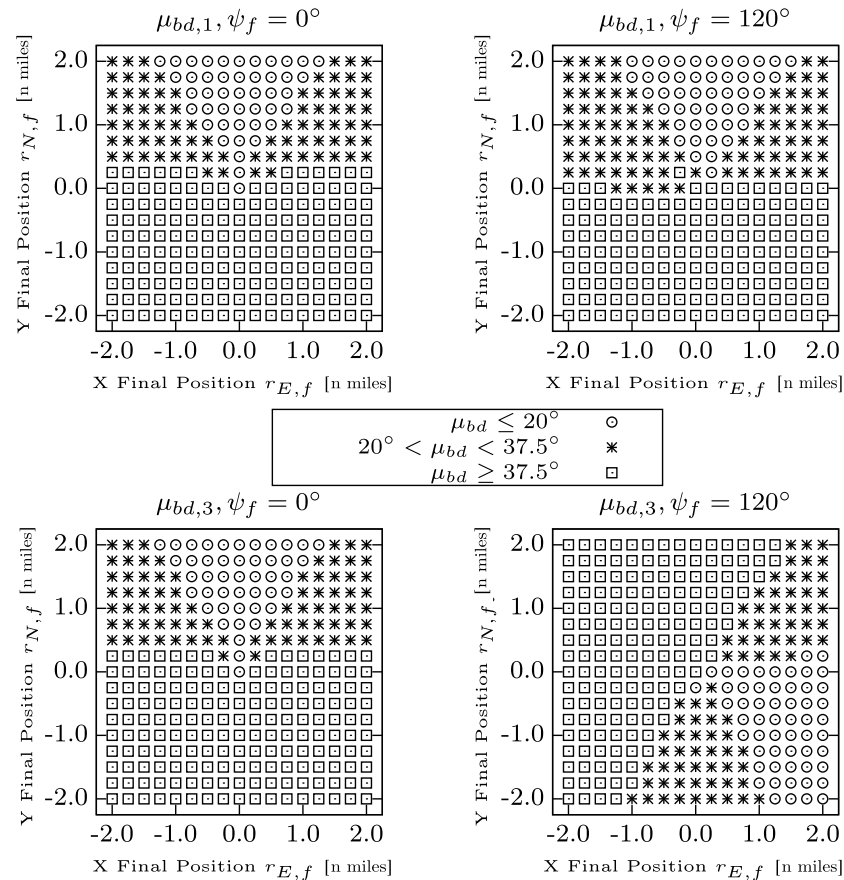

10 Segment 1 and segment 3 best-turn Dubins glider bank angles for different final positions and headings.
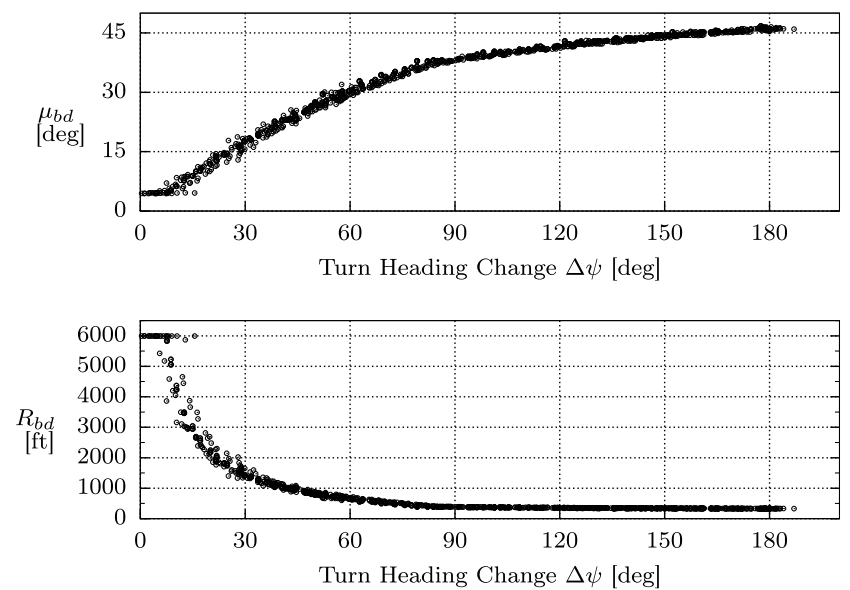

Fig. 11 Best-turn Dubins glider bank and turn radius $\left(\gamma_{\mathrm{bd}}, \boldsymbol{R}_{\mathrm{bd}}\right)$ as a function of total heading change.

Figure 10 presents ranges of best-turn Dubins glider bank angles for each solution computed with Algorithm 2. The upper and lower sets of the two graphs relate to the first and third segments of the trajectories respectively. The three intervals of bank angles are chosen to represent the best-turn Dubins glider flight-path angle close to $\gamma_{\mathrm{bg}}, \gamma_{C L \max }$, and intermediate values as per Fig. 5 . Figure 10 shows that values for optimal bank and flight-path angles vary according to the desired final position radial from the origin and that the orientation of such radials is closely related to the initial and final headings.

These results indicate that the heading change for each turning segment plays a significant role in determining the best-turn Dubins glider bank and turn radius. To investigate further, Fig. 11 presents the optimal $\mu_{\mathrm{bd}}$ and $R_{\mathrm{bd}}$ different heading changes $\Delta \psi$ using the best-turn turning segments. All the turning segments of Fig. 10 with final points at a distance greater than $0.35 \mathrm{n}$ mile $\approx 2127 \mathrm{ft}$ from the initial point are plotted in Fig. 11. Observe that, for these longer distances, the best-turn bank angle $\mu_{\text {bd }}$ increases and the optimum turn radius $R_{\text {bd }}$ decreases as the turning heading change increases. Closer initial
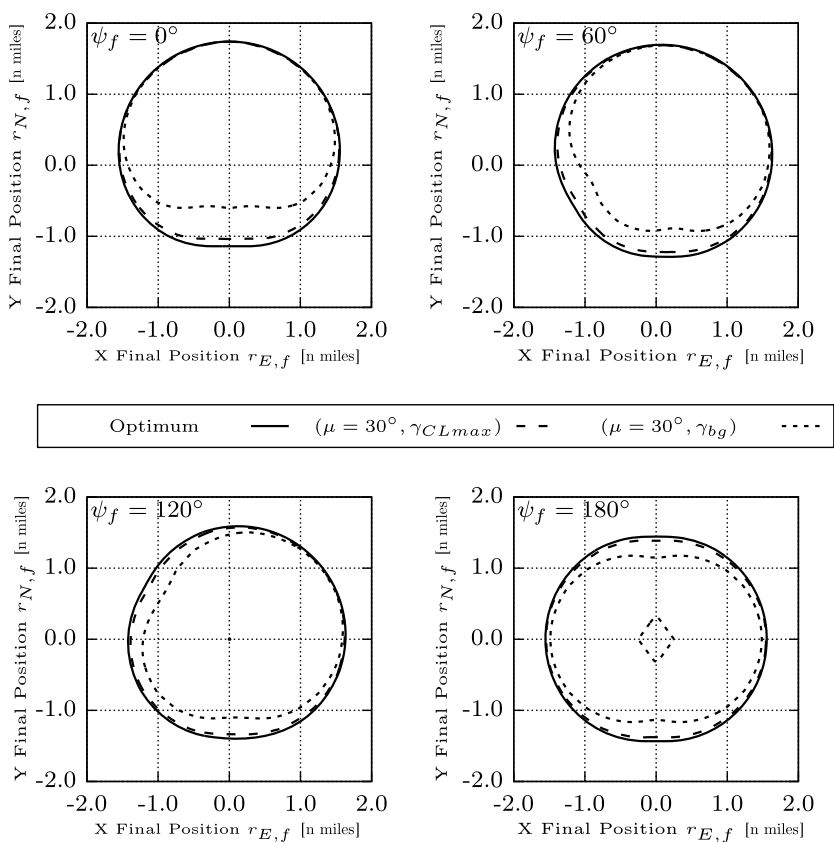

Fig. 12 Contour plots of positions with final altitude loss less than $1000 \mathrm{ft}$ with comparison between best-turn $\left(\gamma_{\mathrm{bd}}, \mu_{\mathrm{bd}}\right)$, best-glide $\left(\gamma_{\mathrm{bg}}\right.$, $\boldsymbol{\mu}=30 \mathrm{deg}$, and minimum flight-path angle $\left(\gamma_{C L \text { max }}, \boldsymbol{\mu}=30 \mathrm{deg}\right)$ strategies. 

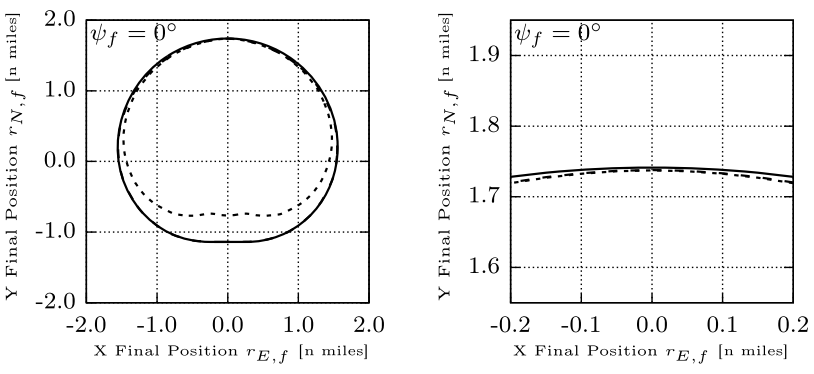

\begin{tabular}{|llll|}
\hline Optimum & $-\left(\mu=45^{\circ}, \gamma_{C L \max }\right)-\cdots$ & $\left(\mu=45^{\circ}, \gamma_{b g}\right)$ & $\cdots$ \\
\hline
\end{tabular}
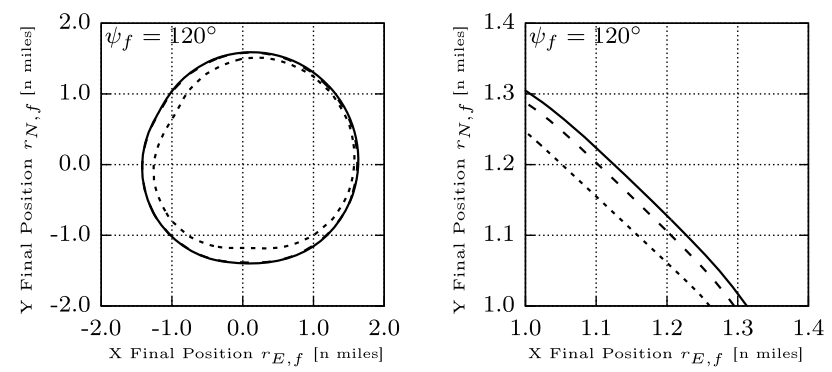

Fig. 13 Contour plots of positions with final altitude loss less than $1000 \mathrm{ft}$ with comparison between best-turn $\left(\gamma_{\mathrm{bd}}, \mu_{\mathrm{bd}}\right)$, best-glide $\left(\gamma_{\mathrm{bg}}\right.$, $\mu=45 \mathrm{deg})$, and best-heading-change $\left(\gamma_{b \psi}=\gamma_{C L \text { max }}, \mu_{b \psi}=45 \mathrm{deg}\right)$ strategies.

and final positions result in trajectories with best-turn bank angle and turn radius a function of traversal distance and heading change.

Also in Fig. 11, note that the relationship between the best-turn bank angle and the turn radius with respect to the heading change is independent of the turning segment and Dubins path type given a final position sufficiently distant from the initial position. Plateaus associated with low values of heading change are explained by the test turn radius upper limit.

To study the impact of choosing a single $(\gamma, \mu)$, Figs. 12 and 13 present a comparison of final positions with an altitude loss of less than $1000 \mathrm{ft}$ for the best turn versus $\mu=30 \mathrm{deg}$ and $\mu=45 \mathrm{deg}$ fixed bank angle cases, respectively. Flight-path angles corresponding to $\gamma_{\mathrm{bg}}$ and $\gamma_{C L \max }$ are used. For Fig. $13,\left(\gamma_{C L \max }, \mu=45 \mathrm{deg}\right)$ is approximately $\left(\gamma_{b \psi}, \mu_{b \psi}\right)$, as discussed in Sec. II.B, with results shown for different final headings $\psi_{f}$.

Observe that $\gamma_{\mathrm{bg}}$ produces worse results than $\gamma_{C L \max }$ with large heading changes. Moreover, when $\mu=30 \mathrm{deg}$ and $\psi_{f}=120 \mathrm{deg}$, some regions close to the initial position require more than $1000 \mathrm{ft}$ of altitude loss. Figure 13 shows that the appropriate choice of $\mu=$ $45 \mathrm{deg}$ and $\gamma_{C L \max }$ provides a near-optimal solution in most conditions.

For trajectories with small heading changes, this $(\gamma, \mu)$ pair is not optimal, as shown by the right column plots zoomed in to areas of interest in Fig. 13. Nevertheless, the need for a zoomed-in image to show differences supports the argument that $\left(\mu=45 \mathrm{deg}, \gamma_{C L \max }\right)$ is sufficiently close to the optimal trajectory, even for cases with small required heading changes over which little altitude is lost.

\section{Conclusions}

The three-dimensional Dubins path-planning literature has traditionally assumed horizontal and vertical motion profiles could be decoupled. For no-thrust or glider fixed-wing aircraft, such coupling could play a significant role. This paper showed how the optimal solutions for both footprint and Dubins glider problems adopted bank and flight-path angle pairs that varied between the two extrema, best-glide and $C_{L \max }$, according to required heading change. For the no-wind case, the maximum range was obtained with a maximum flight-path angle for a given radius. Moreover, if restricted to a single bank and flight-path angle, the results showed that a bank of $45 \mathrm{deg}$ with a flight-path angle to achieve $C_{L \max }$ was close to optimal in most scenarios. The methods to construct sequences of steady flight segments with best turns presented in this paper generalized to any fixed-wing aircraft configuration of any size and propulsion configuration because the only configuration assumptions were no-thrust and standard fixed-wing aerodynamics.

\section{Acknowledgments}

The first author is supported in part by Coordenação de Aperfeiçoamento de Pessoal de Nível Superior-Brazil through the Science Without Borders Program, process number BEX 19130/120 . This work does not reflect official positions of National Civil Aviation Agency-Brazil.

\section{References}

[1] Dubins, L. E., "On Curves of Minimal Length with a Constraint on Average Curvature, and with Prescribed Initial and Terminal Positions and Tangents," American Journal of Mathematics, Vol. 79, No. 3, 1957, pp. 497-516. doi: $10.2307 / 2372560$

[2] Chitsaz, H., and LaValle, S. M., "Time-Optimal Paths for a Dubins Airplane," 46th IEEE Conference on Decision and Control, IEEE Publ., Piscataway, NJ, 2007, pp. 2379-2384. doi:10.1109/CDC.2007.4434966

[3] Atkins, E. M., Portillo, I. A., and Strube, M. J., "Emergency Flight Planning Applied to Total Loss of Thrust," Journal of Aircraft, Vol. 43, No. 4, 2006, pp. 1205-1216. doi:10.2514/1.18816

[4] Coombes, M., Chen, W.-H., and Render, P., "Reachability Analysis of Landing Sites for Forced Landing of a UAS," Journal of Intelligent and Robotic Systems, Vol. 73, Nos. 1-4, 2014, pp. 635-653. doi:10.1007/s10846-013-9920-9

[5] Rademacher, B. J., Lu, P., Strahan, A. L., and Cerimele, C. J., "In-Flight Trajectory Planning and Guidance for Autonomous Parafoils," Journal of Guidance, Control, and Dynamics, Vol. 32, No. 6, 2009, pp. 1697-1712. doi: $10.2514 / 1.44862$

[6] Rogers, D. F., "Possible 'Impossible' Turn," Journal of Aircraft, Vol. 32, No. 2, 1995, pp. 392-397. doi: $10.2514 / 3.46728$

[7] Lowry, J. T., "Glide Performance," Performance of Light Aircraft, AIAA, Reston, VA, 1999, pp. 277-316, Chap. 9.

[8] Adler, A., Bar-Gill, A., and Shimkin, N., "Optimal Flight Paths for Engine-Out Emergency Landing," 24th Chinese Control and Decision Conference (CCDC), IEEE Publ., Piscataway, NJ, 2012, pp. 2908-2915. doi:10.1109/CCDC.2012.6244461

[9] Stephan, J., and Fichter, W., "Fast Generation of Landing Paths for Fixed-Wing Aircraft with Thrust Failure," AIAA Guidance, Navigation, and Control Conference, AIAA Paper 2016-1874, 2016, pp. 1-12. doi:10.2514/6.2016-1874

[10] Cockayne, E., and Hall, G., "Plane Motion of a Particle Subject to Curvature Constraints," SIAM Journal on Control, Vol. 13, No. 1, 1975, pp. 197-220. doi:10.1137/0313012

[11] Thomaschewski, B., "Workspaces of Continuous Robotic Manipulators," Ph.D. Dissertation, Technische Univ. Ilmenau, Ilmenau, Germany, 2002, http://d-nb.info/974856967 [retrieved 2016].

[12] Bakolas, E., Zhao, Y., and Tsiotras, P., "Initial Guess Generation for Aircraft Landing Trajectory Optimization," AIAA Guidance, Navigation, and Control Conference, AIAA Paper 2011-6689, 2011, pp. 8-0. doi:10.2514/6.2011-6689

[13] McClamroch, N. H., Stea dy Aircraft Flight and Performance, Princeton Univ. Press, Princeton, NJ, 2011, Chaps. 3.8, 6, 10.

[14] Pamadi, B. N., "Turning Flight," Performance, Stability, Dynamics, and Control of Airplanes, 2nd ed., AIAA Education Series, AIAA, Reston, VA, 2004, pp. 122-146, Chap. 2.8.

[15] U.S. National Aeronautics, and Space Administration (NASA), "U.S. Standard Atmosphere 1976," NASA TM-X-74335, Oct. 1976, pp. 1-20.

[16] “Type Certificate Data Sheet," Rev. 79, Dept. of Transportation, Federal Aviation Administration, No. 3A12, 2009.

[17] Sussmann, H. J., "Shortest 3-Dimensional Paths with a Prescribed Curvature Bound," Proceedings of the 34th IEEE Conference on Decision and Control, Vol. 4, IEEE Publ., Piscataway, NJ, 1995, pp. 3306-3312. doi:10.1109/CDC.1995.478997

[18] Hota, S., and Ghose, D., "Optimal Path Planning for an Aerial Vehicle in 3D Space," 49th IEEE Conference on Decision and Control (CDC), 
IEEE Publ., Piscataway, NJ, 2010, pp. 4902-4907. doi: $10.1109 / C D C .2010 .5717246$

[19] Owen, M., Beard, R. W., and McLain, T. W., "Implementing Dubins Airplane Paths on Fixed-Wing UAVs*," Handbook of Unmanned Aerial Vehicles, Springer, New York, 2014, pp. 1677-1701. doi:10.1007/978-90-481-9707-1_120

[20] Shen, Y.-F., Rahman, Z.-U., Krusienski, D., and Li, J., "A Vision-Based Automatic Safe Landing-Site Detection System," IEEE Transactions on Aerospace and Electronic Systems, Vol. 49, No. 1, 2013, pp. 294-311. doi:10.1109/TAES.2013.6404104

[21] Meuleau, N., Plaunt, C., Smith, D. E., and Smith, T. B., "An Emergency Landing Planner for Damaged Aircraft," Proceedings of the 21st
Innovative Applications of Artificial Intelligence Conference, AAAI Press, Menlo Park, CA, 2009, pp. 114-121.

[22] Siegel, D., "Development of an Autoland System for General Aviation Aircraft," M.S. Thesis, Massachusetts Inst. of Technology, Cambridge, MA, 2011.

[23] Bui, X.-N., Boissonnat, J.-D., Soueres, P., and Laumond, J.-P., "Shortest Path Synthesis for Dubins Non-Holonomic Robot," Proceedings of the 1994 IEEE International Conference on Robotics and Automation, IEEE Publ., Piscataway, NJ, 1994, pp. 2-7.

doi:10.1109/ROBOT.1994.351019 